\title{
Adaptive Thresholding with Inverted Triangular Area for Real-Time Detection of the Heart Rate from Photoplethysmogram Traces on a Smartphone
}

\author{
Wen Jun Jiang ${ }^{1}$, Peter Wittek ${ }^{2}$, Li Zhao ${ }^{1}$ and Shi Chao Gao ${ }^{1}$
}

\begin{abstract}
Photoplethysmogram (PPG) signals acquired by smartphone cameras are weaker than those acquired by dedicated pulse oximeters. Furthermore, the signals have lower sampling rates, have notches in the waveform and are more severely affected by baseline drift, leading to specific morphological characteristics. This paper introduces a new feature, the inverted triangular area, to address these specific characteristics. The new feature enables real-time adaptive waveform detection using an algorithm of linear time complexity. It can also recognize notches in the waveform and it is inherently robust to baseline drift. An implementation of the algorithm on Android is available for free download. We collected data from 24 volunteers and compared our algorithm in peak detection with two competing algorithms designed for PPG signals, IncrementalMerge Segmentation (IMS) and Adaptive Thresholding (ADT). A sensitivity of $98.0 \%$ and a positive predictive value of $98.8 \%$ were obtained, which were $7.7 \%$ higher than the IMS algorithm in sensitivity, and $8.3 \%$ higher than the ADT algorithm in positive predictive value. The experimental results confirmed the applicability of the proposed method.

Index Terms-Photoplethysmogram, PPG, peak detection, waveform detection, real time, adaptive threshold, smartphone
\end{abstract}

\section{INTRODUCTION}

Recent developments focus on non-invasive measurements of cardiac activity that cause the least amount of discomfort to the patients. Photoplethysmogram (PPG) is an optical measurement at the surface of skin. It is composed of two low-cost opto-electronic components [1]: a light source illuminating the skin and a photodiode measuring the amount of light. Depending on the placement of the photodiode, either the transmitted or reflected light is measured.

The intensity of the light reflects the change in vascular blood volume, which is based on the Beer-Lambert law. According to this law, the intensity of the transmitted light is inversely proportional to the volume of the blood present in the tissue. Moreover, the light intensity reaches its peak when the arterial blood volume is at its lowest during diastole. Accordingly, the light intensity reaches its valley when the arterial blood volume is at its highest during systole [2].

Traditionally PPG signals are obtained by a pulse oximeter. Although a pulse oximeter is affordable and compact, it is seldom purchased or used by ordinary people. Mobile phones are a solution to this situation. They pervade all aspects of daily life, owing to their portability, usefulness, convenience, and relative low-cost [3]. The latest advances

\footnotetext{
${ }^{1}$ W.J. Jiang, L. Zhao, and S.C. Gao are with the Tsinghua University.

${ }^{2} \mathrm{P}$. Wittek is with the University of Borås.
}

prove the feasibility of commodity mobile phones as devices for obtaining PPG signals for heart rate measurement with a finger placed on the camera lens, leading to comparable results with pulse oximeters [4]. PPG signals obtained this way offer other physiological parameters, such as breathing rate and blood oxygen saturation [5], [6], [7]. The primary advantage is that users do not need specialized hardware.

Detecting the basic characteristics, such as the foot and the peak of the PPG waveform, is an important step in studying the signal. An efficient algorithm is incrementalmerge segmentation (IMS) [8], which takes advantage of the obvious difference in amplitude between primary peaks and notches in the wave. A competing algorithm, adaptive threshold detection (ADT) [9], is designed with a virtual threshold which is controlled by original PPG waveform amplitude, using previous pulse interval to estimate the position of a new peak.

However, PPG signals acquired by smartphone cameras lead to a range of problems: the frame rate is low, unusual artifacts - noise - enter the signal, and the waveform contains an obvious baseline drift. Accurate estimation of the heart rate on the phone itself still calls for further research.

We propose an algorithm using a new measure, the inverted triangular area (ITA), for this scenario, which is able to overcome the notches in the PPG waveform and it is also robust to baseline drift.

\section{Proposed Method}

\section{A. Inverted triangular area}

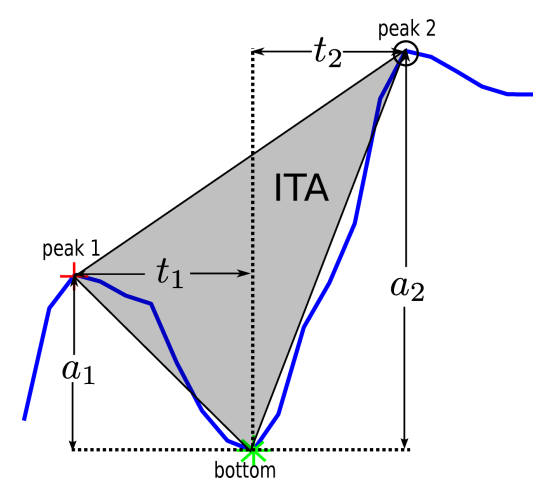

Fig. 1. Defining characteristics of a signal: peak 1 (red cross), bottom (green asterix), and peak 2 (black circle) compose a unit. The parameters $a_{1}, a_{2}, t_{1}, t_{2}$, and the ITA describe the unit. 
A period of wave is composed of a falling edge and a subsequent rising edge. This period of wave is composed of two local maxima (peaks 1 and 2 in Figure 1) and a local minimum (bottom in Figure 1). We call this a unit and use five parameters to describe it: $a_{1}, a_{2}, t_{1}, t_{2}$, and ITA, as shown in Figure 1. The parameters $a_{1}$ and $t_{1}$ are the vertical distance and horizontal distance between the bottom and peak 1 , while $a_{2}$ and $t_{2}$ are the distance between the bottom and peak 2. We define ITA as the area of the triangle peak 1, peak 2 and bottom and it can be calculated through the following formula:

$$
\begin{aligned}
\text { ITA } & =\frac{1}{2}\left(a_{1}+a_{2}\right)\left(t_{1}+t_{2}\right)-\frac{1}{2} a_{1} t_{1}-\frac{1}{2} a_{2} t_{2} \\
& =\frac{1}{2}\left(a_{1} t_{2}+a_{2} t_{1}\right) .
\end{aligned}
$$

We also note that several successive units can be merged to a new unit, whose peak 1 is that of the first unit, whose peak 2 is that of the last unit, and whose bottom is the minimum bottom among the these units.

Time duration can distinguish primary peaks from notches in the wave. But it doesn't work when these notches appear near the bottom of the signal. However, ITA can deal with this situation, because the small rising edges of these notches make their ITAs small, as shown in Figure 2.

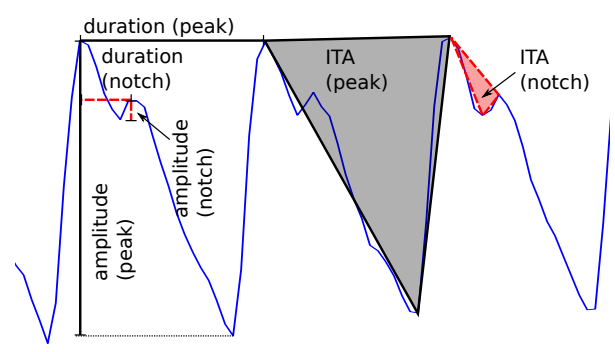

Fig. 2. The amplitude, duration, and ITA for the primary peak and the notch. The red, dashed lines indicate the components of the notch, whereas the solid black color indicates those of the primary peak.

Furthermore, subtle movement of the finger on the lens add baseline drift to the amplitude of the PPG signal. This kind of noise is irregular in itself, but in a short period of time, like the period between two neighboring pulses, we model it as a linear noise with slope $\theta\left(-90^{\circ}<\theta<90^{\circ}\right)$, which comes from the idea that baseline drift can be fit by polynomial [10]. Unlike the sensitivity of the amplitude of the rising edge to baseline drift in IMS, ITA is unaffected by it. If we add a linear drift to the raw signal with a slope $\theta$ as that in Figure 3, the 5 parameters change as follows: $t_{1}^{\prime}=t_{1}, t_{2}^{\prime}=t_{2}, a_{1}^{\prime}=a_{1}-\theta t_{1}, a_{2}^{\prime}=a_{2}+\theta t_{2}$.

$$
\begin{aligned}
\mathrm{ITA}^{\prime} & =\frac{1}{2}\left(a_{1}^{\prime} t_{2}^{\prime}+a_{2}^{\prime} t_{1}^{\prime}\right) \\
& =\frac{1}{2}\left(\left(a_{1}-\theta t_{1}\right) t_{2}+\left(a_{2}+\theta t_{1}\right) t_{1}\right) \\
& =\frac{1}{2}\left(a_{1} t_{2}+a_{2} t_{1}\right)=\mathrm{ITA} .
\end{aligned}
$$

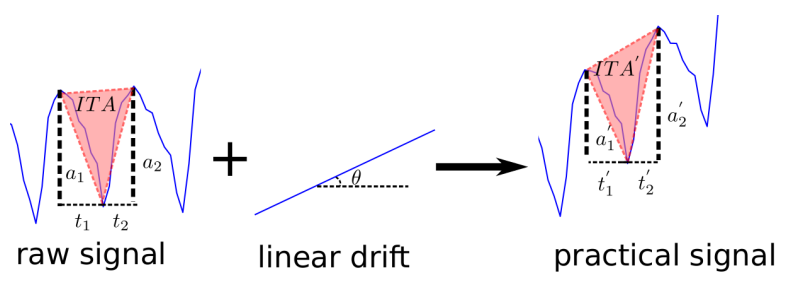

Fig. 3. When a raw PPG signal has a linear drift with a slope $\theta$, its parameters changed from $a_{1}, a_{2}, t_{1}, t_{2}, I T A$ to $a_{1}^{\prime}, a_{2}^{\prime}, t_{1}^{\prime}, t_{2}^{\prime}, I T A^{\prime}$.

The result shows that the rising edge amplitude $\left(a_{2}\right.$ in Figure 3 ) changes according to the slope of the drift, from $a_{2}$ to $a_{2}+\theta t_{2}$. In contrast, ITA will not change.

The above two characteristics of ITA make it suitable for an accurate and robust peak detection algorithm on PPG signals obtained by a smartphone.

\section{B. Adaptive Waveform Detection on a Smartphone}

The workflow is outlined in Figure 4. After preprocessing, we add units from the PPG signal to the unit list, merge all the units in the unit list, and detect the primary peaks through comparing ITA with the threshold. We explain the procedure in the flow chart below:

1) Step 1: preprocessing: Interpolating and smoothing the signal. Obtaining the threshold value if there is no threshold.

2) Step 2: get_unit(): Detecting a falling edge and a subsequent rising edge in the wave to construct a unit. Adding the unit to the unit list.

3) Step 3: merge_unit(): In the unit list, using peak 1 of the first unit, peak 2 of the last unit and the minimum bottom among these units to construct a new unit. Calculating $a_{1}, t_{1}, a_{2}, t_{2}$, and ITA by these three vertices.

4) Step 4: $\operatorname{detect}()$ : Comparing the ITA of the unit with the threshold, deciding whether to accept the waveform, updating the threshold, and returning to step 1 until reaching the end of the signal.

An implementation is available for download ${ }^{1}$. In what follows, we further detail the processing steps.

\section{Interpolating and Smoothing}

We first check whether the finger is correctly placed on the camera lens by the brightness and standard deviation of RGB channels [11]. When the finger is correctly placed, we record the mean brightness of each frame as PPG signal. Then we interpolate the collected signal to the average sampling rate in the first 5 seconds and only use a 3-point moving average filter to smooth it in order not to modify the waveform [10].

\section{Obtaining the Threshold Value}

We define the heart rate range between 40 and $200 \mathrm{bpm}$. This translates to a time interval between 0.3 seconds and 1.5 seconds for the period between two peaks of an acceptable

\footnotetext{
${ }^{1}$ https://play.google.com/store/apps/details?id= com.kiwihealthcare. cardiacfinger
} 


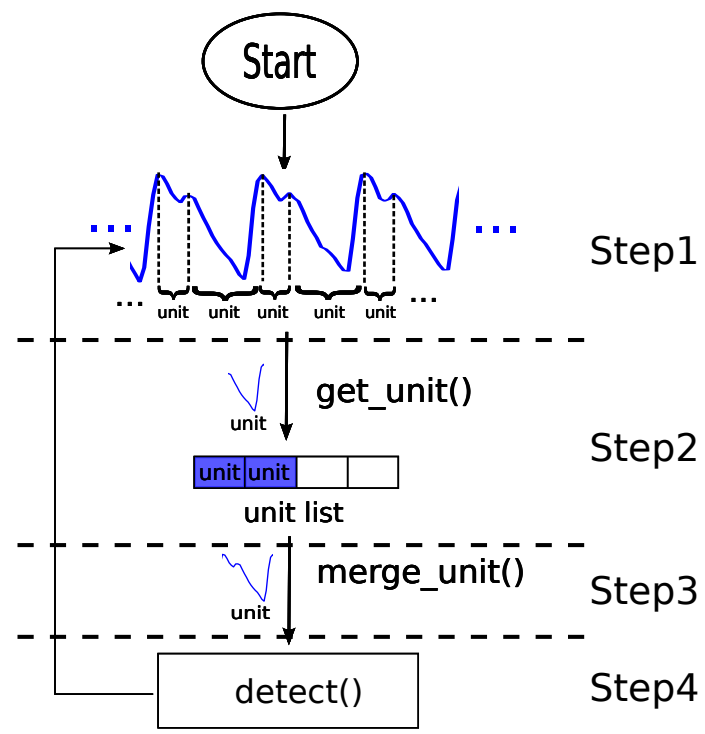

Fig. 4. The core of the detecting loop. We divide the PPG signal by local maxima. In each round, two consecutive maxima and a local minimum constitute a unit obtained by get_unit(). The unit leaving from the function merge_unit() is different: it is merged from all the individual units in the unit list, so its shape is different. It is then compared with the threshold to detect whether the unit is accepted. After that, we begin the next round.

unit. In order to find out peak 1 and peak 2, we first choose a time window of 3 seconds and find out the rising edge with the maximum amplitude within it as the rising edge of peak 1, whose amplitude is noted as $a m p_{1}$. Then we move the time window forward to peak 1 and find out another rising edge in the window as the rising edge of peak2, whose amplitude is noted as $a m p_{2}$, satisfying $a m p_{1}<2 a m p_{2}, a m p_{2}<2 a m p_{1}$ and the time interval restriction. If we find out such rising edges, we use the waveform between them to construct a unit and use its ITA as threshold. If we fail, we select the maximum rising edge in the current window as the rising edge of peak 1 and repeat the the same procedure until we get threshold.

\section{E. Waveform Detection}

In this step, we use the unit merged from the unit list and check the time interval. If the time interval is acceptable, we compare the current ITA with the threshold. (Algorithm 1). If the ITA is in the range [0.4threshold, 2threshold], we update the threshold with the formula:

$$
\text { threshold }=\alpha \text { threshold }+(1-\alpha) \operatorname{ITA}(\text { unit }) \text {. }
$$

If not, we update the threshold with the formula:

$$
\text { threshold }=\beta \text { threshold }+(1-\beta) \operatorname{ITA}(\text { unit }) .
$$

Here $\alpha$ and $\beta$ are both adaptive parameters. In our experiments, we set $\alpha$ as 0.75 and set $\beta$ as 0.9 . Usually we set $\alpha$ and $\beta$ higher than 0.5 and $\beta$ higher than $\alpha$ because we want normal values contribute more to the threshold. We continue this procedure until we get to the end of the signal.

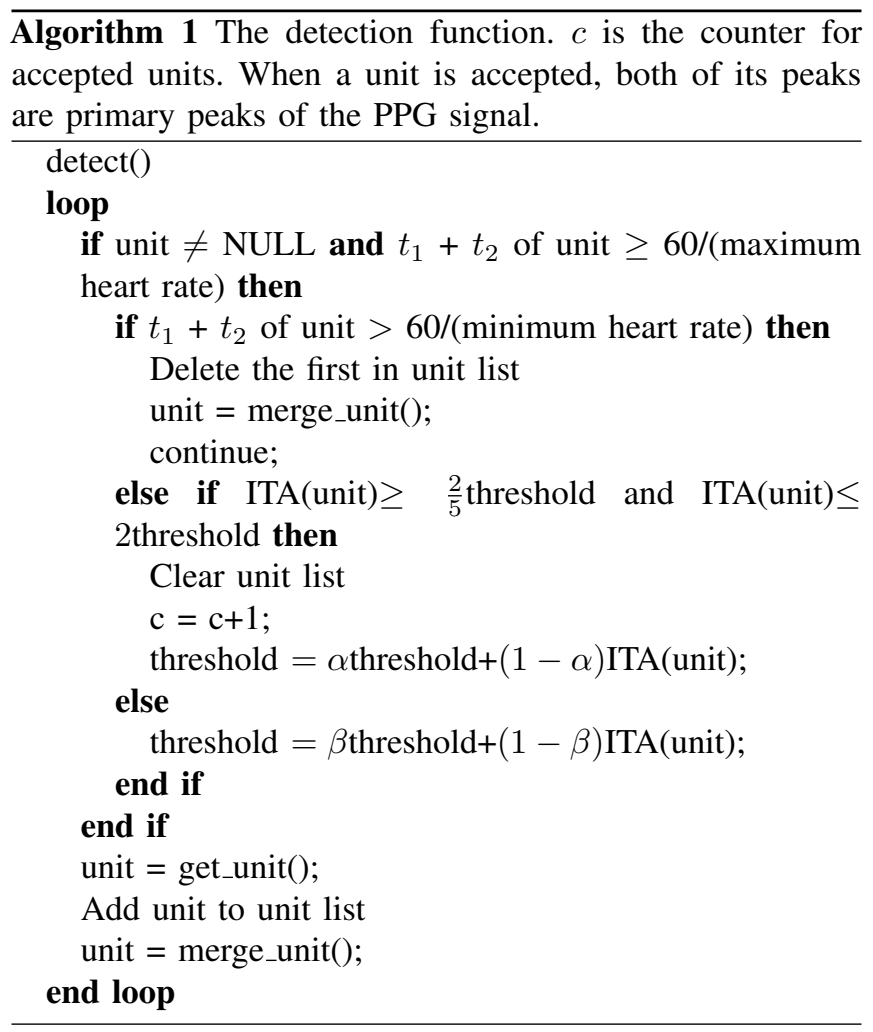

\section{EXPERIMENTAL RESULTS}

\section{A. Data Collection}

We took samples from 27 participants, 16 males and 11 females. During the experiment, the subjects were seated. A pulse oximeter was placed on the right index finger. The participants held the phone in their left hand, placing the left index finger on the camera lens. A custom-developed application on the phone collected the data simultaneously by the smartphone and the pulse oximeter. Among the 27 subjects, the recorded pulse oximeter traces of a male and two female participants were deformed, the finger was not properly placed in the device. Their data were removed, leading to a sample of 24 records.

We used a Motorola Milestone phone for all subjects to acquire the PPG trace. The phone ran Android 4.1. We used a pulse oximeter Etcomm HC-801 as the reference source for the PPG signal. It connects and sends data to the smartphone via Bluetooth version 2.0 protocol. Its sampling rate is $20 \mathrm{~Hz}$. The phone received data from the pulse oximeter using the standard Android Bluetooth API while recording the trace with its camera.

We collected a 60 by 60 pixel average region of a video signal at each frame at roughly 13 frame per second. The original picture format was YUV420sp, we recorded the time stamp and the average value of $\mathrm{Y}$ as time and amplitude of PPG signal.

Through recording PPG signals simultaneously from the smartphone and the pulse oximeter in an Android application, we could synchronize the beginning of the recording. We obtained pulse signals from both the smartphone and the 
pulse oximeter for a total of 120 seconds. Then we labeled the primary peaks of traces from mobile phone through comparing them with the traces from pulse oximeter.

\section{B. Analysis}

We benchmarked the effectiveness of our algorithm against two other peak detection algorithms, ADT [9] and IMS [8] on the traces from mobile phone. A beat-to-beat comparison was applied between the output of three algorithms and the labeled primary peaks. The first ten seconds of each sample were considered the training process and were not included in the comparison. Time deviation within $0.2 \mathrm{~s}$ between beats was accepted. We recorded two parameters for each algorithm:

- Failed detection: the number of labeled primary peaks not found by the algorithm.

- False positive: the number of primary peaks found by the algorithm but not labelled.

\begin{tabular}{cccc|ccc} 
Number & \multicolumn{3}{c}{ Failed detection } & \multicolumn{3}{c}{ False positive } \\
of peaks & ITA & IMS & ADT & ITA & IMS & ADT \\
\hline 3120 & 63 & 303 & 77 & 37 & 26 & 319 \\
\hline \multicolumn{4}{c}{ TABLE I } \\
\hline \multicolumn{4}{c}{}
\end{tabular}

THE ACCURACY OF PEAK DETECTION.

To compare the accuracy of peak detection, we used two criteria: sensitivity (SE) and positive predicted value (PPV). They are defined as follows (a hash mark indicates 'number of'):

$$
\begin{aligned}
S E= & \frac{\# \text { primary peaks }-\# \text { failed detections }}{\text { \#primary peaks }} ; \\
P P V= & (\text { \#primary peaks }-\# \text { failed detections }) / \\
& \text { (\#primary peaks }- \text { \#false detections }+ \\
& \text { \#false positives }) .
\end{aligned}
$$

For an overview of the results, refer to Table I. The average SE was $98.0 \%, 90.3 \%$, and $97.5 \%$ for ITA, IMS, and ADT, respectively. In the same order, the average PPV was $98.8 \%$, $99.1 \%$, and $90.5 \%$.

ADT had a close SE to our algorithm, but a relatively low PPV. This comes from the design of the algorithm. ADT was designed for PPG signals without baseline drift, so its accuracy depended on whether the baseline drift was apparent and how well this could be removed. When the PPG signal was strong, which meant the noise was relatively weak, ADT performed well. But when the PPG signal was weak, like the low perfusion problem, ADT could not address it well [9].

As for the IMS algorithm, since the difference of the rising edge amplitude between the primary peak and the notches was obvious, its PPV was the highest. However, because of the influence of baseline drift, the amplitude between pulses was unstable, and some pulses were not recognized when baseline drift changed.

In contrast, our algorithm got a high SE as well as a high PPV, which was mainly due to the robustness of the inverted triangular area.
Finally, we evaluate the effectiveness of our algorithm in mean heart rate estimation. Heart rate is the reciprocal of time intervals between two neighboring peaks. After we get units, $t_{1}+t_{2}$ is the instantaneous time interval and we use the reciprocal of mean time intervals to estimate mean heart rate. Comparing the results from the pulse oximeter, our estimation of mean heart rate on smartphone get a relative accuracy of $98.6 \%$ in average.

\section{CONCLUSION}

The core idea of our algorithm is to regard the PPG waveform as a chain of elementary units. We aimed to solve two problems occurred in the PPG waveform from a mobile phone: one is the notches in the waveform, the other is the effect of baseline drift. Introducing a new feature, the inverted triangular area, we demonstrated the accuracy of our algorithm compared to two competing method that also have the potential for real-time detection on a smartphone. There are still other forms of distortions that cannot be solved by our method. For example, sometimes the amplitudes of primary peaks are too low to be distinguished from noise. To ensure high accuracy in the estimate, the phone also must estimate the quality of the waveform and provide real-time feedback to users to keep the right gesture.

\section{REFERENCES}

[1] J. Allen, "Photoplethysmography and its application in clinical physiological measurement," Physiol. Meas., vol. 28, no. 3, pp. R1-R39, 2007.

[2] J. G. Webster, Design of pulse oximeters. CRC Press, 2002.

[3] L. Liu and J. Liu, "Biomedical sensor technologies on the platform of mobile phones," Frontiers of Mechanical Engineering, vol. 6, no. 2, pp. 160-175, 2011.

[4] P. Pelegris, K. Banitsas, T. Orbach, and K. Marias, "A novel method to detect heart beat rate using a mobile phone," in Proc. of EMBC-10, 32nd Ann. Int. Conf. of the IEEE Engineering in Medicine and Biology Society, Buenos Aires, Argentina, August 2010, pp. 5488-5491.

[5] J. Lee and K. Chon, "Respiratory rate extraction via an autoregressive model using the optimal parameter search criterion," Ann. Biomed. Eng., vol. 38, no. 10, pp. 3218-3225, 2010.

[6] C. G. Scully, J. Lee, J. Meyer, A. M. Gorbach, D. Granquist-Fraser, Y. Mendelson, and K. H. Chon, "Physiological parameter monitoring from optical recordings with a mobile phone," IEEE Trans. Biomed. Eng., vol. 59, no. 2, pp. 303-306, 2012.

[7] Y. Kurylyak, F. Lamonaca, and D. Grimaldi, "Smartphone based photoplethysmogram measurement," in Digital Image and Signal Processing for Measurement Systems, R. J. Duro and F. L. Peña, Eds. River Publishers, 2012, pp. 135-164.

[8] W. Karlen, J. M. Ansermino, and G. Dumont, "Adaptive pulse segmentation and artifact detection in photoplethysmography for mobile applications," in Proc. of EMBS-12, 34th Ann. Int. Conf. of the IEEE Engineering in Medicine and Biology Society, 2012, pp. 3131-3134.

[9] H. S. Shin, C. Lee, and M. Lee, "Adaptive threshold method for the peak detection of photoplethysmographic waveform," Comput. Biol. Med., vol. 39, no. 12, pp. 1145-1152, 2009.

[10] M. Kaur, B. Singh et al., "Comparison of different approaches for removal of baseline wander from ecg signal," in Proceedings of the International Conference \& Workshop on Emerging Trends in Technology. ACM, 2011, pp. 1290-1294.

[11] F. Lamonaca, Y. Kurylyak, D. Grimaldi, and V. Spagnuolo, "Reliable pulse rate evaluation by smartphone," in Proc. of MeMeA-12, IEEE Int. Symposium on Medical Measurements and Applications, Budapest, Hungary, May 2012, pp. 1-4. 